\title{
Design of a tunable, room temperature, continuous-wave terahertz source and detector using silicon waveguides
}

\author{
T. Baehr-Jones, ${ }^{1, *}$ M. Hochberg, ${ }^{1,3}$ Richard Soref, ${ }^{2}$ and A. Scherer ${ }^{1}$ \\ ${ }^{1}$ Department of Applied Physics, California Institute of Technology, 1200 E California Boulevard, \\ Pasadena, California 91125 \\ ${ }^{2}$ Air Force Research Laboratory Sensors Directorate, Electromagnetic Technology Division, 80 Scott Drive, Hanscom \\ Air Force Base, Bedford, Massachusetts 01731-2909 \\ ${ }^{3}$ E-mail: hochberg@caltech.edu \\ *Corresponding author: thorolf@caltech.edu
}

Received July 30, 2007; revised December 13, 2007; accepted December 28, 2007; posted January 7, 2008 (Doc. ID 85431); published January 30, 2008

\begin{abstract}
We describe the design of a silicon-based source for radiation in the $0.5-14 \mathrm{THz}$ regime. This new class of devices will permit continuously tunable, milliwatt scale, cw, room temperature operation, a substantial advance over currently available technologies. Our silicon terahertz generator consists of a silicon waveguide for near-infrared radiation, contained within a metal waveguide for terahertz radiation. A nonlinear polymer cladding permits two near-infrared lasers to mix, and through difference-frequency generation produces terahertz output. The small dimensions of the design greatly increase the optical fields, enhancing the nonlinear effect. The design can also be used to detect terahertz radiation. (C) 2008 Optical Society of America OCIS codes: $040.0040,040.6040,060.4080,130.0130,130.2790,130.3120$.
\end{abstract}

\section{INTRODUCTION}

The applications of terahertz $(\mathrm{THz})$ radiation, roughly defined as electromagnetic radiation with a frequency ranging $0.5-10 \mathrm{THz}$, are a topic of active research [1]. They include medical imaging [2], remote sensing [3], chemical detection [4], and tactical imaging [5]. One significant impediment in this field is the expense and complexity associated with $\mathrm{THz}$ sources. Current methods for generating $\mathrm{THz}$ frequencies include using spectrometers to filter blackbody radiation [6], pulsed lasers combined with nonlinear optical materials to exploit difference frequency generation [7,8], quantum cascade lasers [9], and highfrequency microwave mixers [10] among others. Another recent approach has been to combine quantum cascade lasers and difference frequency generation [11]. Some recent work on $\mathrm{THz}$ devices has focused on metamaterials, which may enable switching and control of $\mathrm{THz}$ radiation [12], but this has not yet resulted in significant progress on new $\mathrm{THz}$ sources. All of the aforementioned methods of generating $\mathrm{THz}$ radiation suffer from either very low operating temperatures, lack of tunability or very low power output, or can function only in pulsed mode. The majority of current approaches to $\mathrm{THz}$ generation in fact suffer from several of these problems [13].

Current approaches to using $\chi^{2}$ nonlinearities suffer from low conversion efficiencies [7,8]. This is due in part to the fact that $\mathrm{THz}$ modes must have relatively large lateral dimensions, as well as the fact that nonlinear frequency conversion is inherently less efficient when the output is at lower frequencies. That is why current ap- proaches generally require pulsed lasers. Several recent developments have made possible a new approach to difference frequency generation, however. First, it has become possible to guide optical modes with relatively low loss in small, high-index-contrast, ridge waveguides. Realizations in SOI, for example, achieve $7 \mathrm{~dB} / \mathrm{cm}$ of loss in a $0.5 \times 0.1 \mu \mathrm{m}$ waveguide [14]. Second, nonlinear polymers have become increasingly powerful, with an $r_{33}$, the standard metric of nonlinearity, of $170 \mathrm{pm} / \mathrm{V}$ already demonstrated [15]. It is expected that a further $3 \times$ improvement will be possible, with $r_{33}$ values that will approach $500 \mathrm{pm} / \mathrm{V}$ [16], about 1 order of magnitude stronger than typical nonlinear crystals [17]. While these nonlinear polymers are typically used for electrooptic modulation, we have shown unambiguously that the nonlinearity is ultrafast [18]. When combined with the proper $\mathrm{THz}$ waveguide design, and with the relatively large amounts of laser power that are now available, we will show that practical amounts of $\mathrm{cw} \mathrm{THz}$ radiation can be generated.

\section{THEORETICAL BACKGROUND}

Maxwell's equations can be written for a nonlinear medium as follows [19]:

$$
\left(\begin{array}{cc}
\varepsilon_{0} \partial_{t} \varepsilon & -\nabla \times \\
\nabla \times & \mu_{0} \partial_{t}
\end{array}\right)\left(\begin{array}{c}
\mathbf{E} \\
\mathbf{H}
\end{array}\right)=\left(\begin{array}{c}
-\varepsilon_{0} \partial_{t} \chi_{i j k}^{2} \mathbf{E}_{j} \mathbf{E}_{k} \\
0
\end{array}\right)
$$


Here the subscripts $i, j$, and $k$ represent a summation over the $x, y$, and $z$ components for each vector. We will consider our waveguide to be described by a dielectric function that is continuous in the propagation direction, which we will consider to be the $z$ direction. The inclusion of metals presents no difficulties if the dielectric constant is permitted to approach infinity in some locations. A guided mode will always have the form

$$
c_{i}(z) \psi_{i}(x, y) \exp \left(i \beta_{i} z-i \omega_{i} t\right)
$$

We will neglect the effects of loss for the moment. Physically, the fields are always real. Therefore, the most general form in which a series of propagating waves in a waveguide can be written is

$$
\begin{aligned}
\left(\begin{array}{l}
\mathbf{E} \\
\mathbf{H}
\end{array}\right)= & \sum_{i}\left(c_{i}(z) \psi_{i}(x, y) \exp \left(i \beta_{i} z-i \omega_{i} t\right)\right. \\
& \left.+c_{i}^{*}(z) \psi_{i}^{*}(x, y) \exp \left(-i \beta_{i} z+i \omega_{i} t\right)\right) .
\end{aligned}
$$

Note the $z$ dependence of the mode constant. For a linear material, $c$ will not have any $z$ dependence. But for a nonlinear material, this will not necessarily be true. Putting Eq. (2) in Eq. (1) yields

$$
\begin{gathered}
\sum_{i} \partial_{z} c_{i}(z)\left(\begin{array}{c}
H_{y}(x, y) \\
-H_{x}(x, y) \\
0 \\
-E_{y}(x, y) \\
E_{x}(x, y) \\
0
\end{array}\right) \exp \left(i \beta_{i} z-i \omega_{i} t\right)+\text { c.c. } \\
=-\varepsilon_{0} \partial_{t}\left(\begin{array}{c}
\chi_{x j k}^{2} \mathbf{E}_{j} \mathbf{E}_{k} \\
\chi_{y j k}^{2} \mathbf{E}_{j} \mathbf{E}_{k} \\
\chi_{z j k}^{2} \mathbf{E}_{j} \mathbf{E}_{k} \\
0 \\
0 \\
0 \\
0
\end{array}\right) .
\end{gathered}
$$

Here the mode vector has been expanded to show the individual components. c.c. denotes the complex conjugate of the previous expression. Expanding Eq. (4) yields a series of different equations for different harmonic components. Let us make the discussion more specific. Consider $\omega_{1}$ and $\omega_{2}$ to be two optical beams near $1550 \mathrm{~nm}$. These beams can easily be selected to generate a difference frequency in the THz. For instance, the difference frequency between a beam at 1550 and $1558 \mathrm{~nm}$ is about $1 \mathrm{THz}$. Let $\omega_{3}$ be this difference frequency:

$$
\begin{aligned}
& \left(\begin{array}{c}
H_{3, y} \\
-H_{3, x} \\
0 \\
-E_{3, y} \\
E_{3, x} \\
0
\end{array}\right) \exp \left(i \beta_{3} z-i \omega_{3} t\right) \\
= & -2 \varepsilon_{0}\left(-i \omega_{3}\right) \exp \left(i \beta_{1} z-i \beta_{2} z\right) \exp \left(-i \omega_{3} t\right) c_{1}(z) c_{2}{ }^{*}(z) \\
& \times\left(\begin{array}{c}
\chi_{x j k}^{2} E_{1, j} E_{2, k}^{*} \\
\chi_{y j k}^{2} E_{1, j} E_{2, k}^{*} \\
\chi_{z j k}^{2} E_{1, j} E_{2, k}^{*} \\
0 \\
0 \\
0
\end{array}\right) .
\end{aligned}
$$

Here $E_{1}, E_{2}$ and so forth denote the relevant electric or magnetic field patterns for each mode. It should be noted that Eq. (5) is not quite correct; it is possible that additional modes could exist at $\omega_{3}$, which in principle could be excited as well by the nonlinear perturbation. However, consider that

$$
\begin{gathered}
\int\left(E_{i, x}^{*} E_{i, y}^{*} E_{i, z}^{*} H_{i, x}^{*} H_{i, y}^{*} H_{i, z}^{*}\right)\left(\begin{array}{c}
H_{j, y} \\
-H_{j, x} \\
0 \\
-E_{j, y} \\
E_{j, x} \\
0
\end{array}\right) \mathrm{d} A \\
=\int\left(\mathbf{E}_{i}^{*} \times \mathbf{H}_{j}+\mathbf{E}_{j} \times \mathbf{H}_{i}^{*}\right) \cdot \mathbf{z d} A .
\end{gathered}
$$

It can be shown [20] that any two modes at a given frequency with differing propagation constants are orthogonal under the metric given in Eq. (6). Any degenerate subspace can of course be orthogonalized with the usual methods. As a result, if we assume that there is no degeneracy in the mode set involved in Eq. (5), we can integrate the entire expression across the $x, y$ plane to solve for $\partial_{z} c_{3}$. It's worth noting that if the modal field patterns are normalized so that for each mode, Eq. (6) evaluates to 1, then the corresponding physical mode as found in Eq. (4) has a time-averaged power flow of $1 \mathrm{~W}$. We adopt this convention, and as a result the time-averaged power in a mode in watts is equal to the modulus squared of the corresponding modal amplitude $c_{i}(z)$. Integrating Eq. (5) yields

$$
\begin{aligned}
\partial_{z} c_{3}(z)= & -2 \varepsilon_{0}\left(-i \omega_{3}\right) \exp \left(i \beta_{1} z-i \beta_{2} z-i \beta_{3} z\right) \\
& \times c_{1}(z) c_{2}^{*}(z) \int E_{3, i}^{*} \chi_{i j k}^{2} E_{1, i} E_{2, j}^{*} \mathrm{~d} A .
\end{aligned}
$$

In the event that the propagation constant difference is zero $\left(\beta_{1}-\beta_{2}-\beta_{3}=0\right)$, Eq. (7) can be written in a simpler form. Let us also assume that the only important nonlinear contribution in Eq. (7) stems from the nonlinear moment along a single lateral direction, taken to be $x$ with- 
out loss of generality. Finally, the possibility of loss for mode 3 is added. In this case,

$$
\partial_{z} c_{3}(z)=-2\left(-i \omega_{3}\right) c_{1}(z) c_{2}^{*}(z) \chi_{x x x}^{2} \gamma-\alpha_{3} c_{3}(z),
$$

where the figure of merit $\gamma$ has units of volt-seconds per square meter and is defined to be

$$
\gamma=\varepsilon_{0} \int E_{3, x}^{*} E_{1, x} E_{2, x}^{*} \mathrm{~d} A .
$$

Let us further assume that the two input beams have negligible loss compared to the generated $\mathrm{THz}$ beam, and that only a small fraction of their power is converted to $\mathrm{THz}$ radiation. It is then possible to write down the final amount of power that is generated in mode 3 due to nonlinear conversion from modes 1 and 2 :

$$
P_{3}(L)=\frac{4 \omega_{3}^{2} P_{1} P_{2}\left(\chi_{x x x}^{2} \gamma\right)^{2}}{\alpha_{3}^{2}}\left(1-\exp \left(-\alpha_{3} L\right)\right)^{2} .
$$

We note that the phase matching condition in Eq. (7) can be rewritten as

$$
n_{3}=\frac{n\left(\omega_{1}\right) \omega_{1}-n\left(\omega_{2}\right) \omega_{2}}{\omega_{1}-\omega_{2}} .
$$

We show in this paper that perfect phase matching is attained in a continuous, nonsegmented waveguide by engineering the three effective refractive indices of the composite waveguide.

Typically, electro-optic polymers are characterized by their $r_{33}$ coefficient. This is defined as follows [17]:

$$
\frac{1}{n_{z}^{\prime 2}}-\frac{1}{n_{z}^{2}}=E_{z} r_{33}
$$

That is, the shift in refractive index along the poling axis (taken to be $z$ here) is defined as a function of the product of $r_{33}$ and the driving electric field. This can be represented as a $\chi^{2}$ for small shifts in index as

$$
\chi_{z z z}^{2}=\frac{n_{z}^{4} r_{33}}{2} .
$$

This is true only if the nonlinear effect is not attenuated at higher frequencies. In general, this is often untrue; however, the nonlinear mechanism involved in electrooptic modulation by nonlinear polymers such as these is based on a deformation of an electron orbital and can thus be expected to be ultrafast. Additionally, we have previously demonstrated optical rectification, an ultrafast phenomenon, based on these polymers [18], and our results suggested that the $\chi^{2}$ will be relatively unattenuated at even the optical frequency.

The refractive index of these nonlinear polymers is typically around 1.7 . Therefore, if $500 \mathrm{pm} / \mathrm{V}$ of tuning is obtained, we can expect a $\chi^{2}$ of $2 \times 10^{-9} \mathrm{~m} / \mathrm{V}$.

Before discussing a particular waveguide configuration, it is useful to calculate the order of magnitude of results that might be expected. Provided that a given interacting waveguide geometry achieves the phase-matching condition [Eq. (11)] between the various modes, the governing features will be the areas that the modes are in, their ef- fective indices, and the extent of overlap in the region of the nonlinear material. If the modes are normalized so the moment in Eq. (6) is $1 \mathrm{~W}$, then the average electric field will be approximately

$$
E=\frac{\sqrt{P}}{\sqrt{2 A n}}\left(\frac{\mu_{0}}{\varepsilon_{0}}\right)^{1 / 4} .
$$

Here $P$ is the normalized power, which we set to $1 \mathrm{~W}$ by convention, while $n$ is the effective index of the mode. Given this expression, one can then calculate Eq. (9) as follows:

$$
\gamma=\varepsilon_{0}\left(\frac{\mu_{0}}{\varepsilon_{0}}\right)^{3 / 4} \frac{1}{\sqrt{n_{1} n_{2} n_{3}}} \frac{A_{c}}{\sqrt{A_{1} A_{2} A_{3}}} \frac{1}{2^{3 / 2}} \sqrt{P} .
$$

Here $A_{1}, A_{2}$, and $A_{3}$ are the areas of the relevant modes; $n_{1}, n_{2}$, and $n_{3}$ are the effective indices; and $A_{c}$ is the area where all of the modes and the $\chi^{2}$ material overlap. $P$ is the normalized time-averaged power of all three modes, which is always set to $1 \mathrm{~W}$, included here for clarity of units. With this expression, it is easy to estimate what conversion efficiencies might be approximately obtained for a given interacting waveguide geometry. Let us imagine that we have engineered a situation where $A_{1}=A_{2}$ $=10^{-13} \mathrm{~m}^{2}, A_{3}=84 \times 10^{-12} \mathrm{~m}^{2}$, and $A_{c}=1 \times 10^{-13} \mathrm{~m}^{2}$, and all effective indices are 2. In this case, $\gamma$ will be 8.8 $\times 10^{-4} \mathrm{~V} \mathrm{~s} / \mathrm{m}^{2}$.

As will be shown, confinements of $\mathrm{THz}$ modes to these kinds of area are possible, though the waveguides are lossy. In one instance, the loss near $1 \mathrm{THz}$ is about $25 \mathrm{~dB} / \mathrm{cm}$. In this case, $\alpha_{3}=287 \mathrm{~m}^{-1}$. Assuming that both optical beams are at a power level of $0.5 \mathrm{~W}$, the output power would be $1.5 \mathrm{~mW}$. This level of output power would be reached to within $1 \mathrm{~dB}$ in about $7.5 \mathrm{~mm}$ of device.

\section{PROPOSED DEVICE}

As one will conclude from Section 2, the challenges of designing a silicon terahertz generator break down into three areas: First, designing waveguides that will guide both optical and terahertz radiation, both without crippling losses; second, optimizing the modal overlap with the nonlinear materials to provide efficient difference frequency generation; and third, the waveguides designed so that phase-matching conditions between the $\mathrm{THz}$ and near-IR (NIR) signals are met.

It is well known that silicon can be used to guide radiation in the NIR, and that silicon is relatively transparent in much of the $\mathrm{THz}$ [21]. In addition, because of silicon's high index, silicon waveguides can concentrate optical modes to areas of $1 / 1000$ the size of the modes of ordinary optical fibers and can be evanescently coupled to nonlinear optical polymers [22]. Silicon microscale waveguides can be efficiently coupled both to free space and to fibers [23], and large-scale integrated photonic structures can be made using such waveguides [24,25].

Inexpensive, high-power sources in the range of 1480-1600 nm have recently become widely available, owing to demand from telecommunications applications [26]. Restricting pump sources to this frequency range, the frequencies available as a difference of the two 
sources range from dc to $15 \mathrm{THz}$. High-power lasers are also available in specific bands in the $1300-1480 \mathrm{~nm}$ range, though there are not yet, to our knowledge, single sources that are tunable across the entire range. Based on current laser and erbium-doped fiber amplifier technology, one can expect to obtain approximately $0.5 \mathrm{~W}$ of $\mathrm{cw}$ laser power [27], though this power can be expected to improve in coming years.

Our proposed STG design consists of a silicon core for guiding NIR light, within a metal waveguide for confining $\mathrm{THz}$ radiation; optimal performance would be obtained from copper, but other metals could be used. This basic geometry is shown in Fig. 1. A second-order optical nonlinearity enables the two optical signals to mix and generate a difference frequency, and various dimensions can be optimized in order to achieve phase matching. The entire structure is supported by a micromachined silicon pillar.

The optical waveguide in the STG, shown in Fig. 2, consists of a strip of silicon within a dual-strip metal $\mathrm{THz}$ waveguide. This entire structure is embedded in a cladding of electrooptic polymer [28] with a refractive index of 1.7. This waveguide geometry creates a significant evanescent NIR field, which overlaps with the polymer cladding. Panel A in Fig. 2 shows the waveguide structure and the optical mode overlaid, for the 500-nm-wide wave-

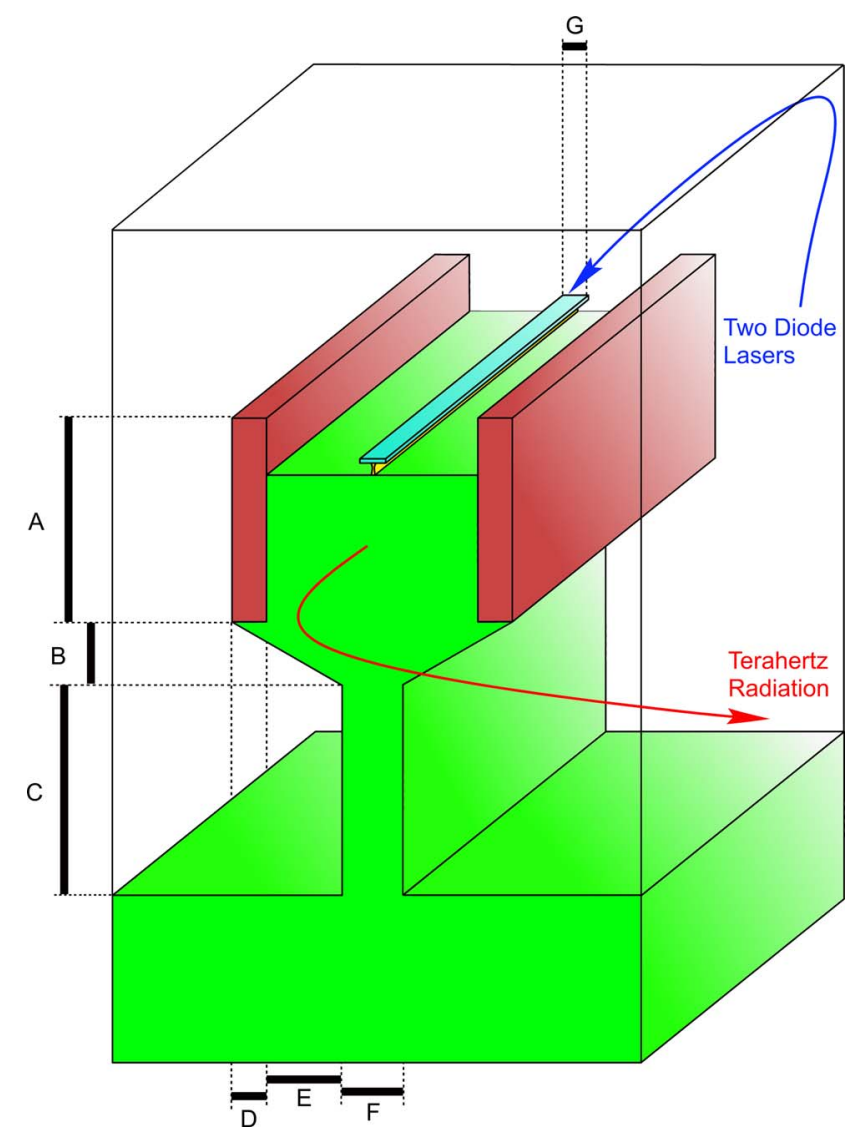

Fig. 1. (Color online) Diagram of the terahertz generation device. An isometric view of the device is shown. The narrow rectangle corresponds to the silicon waveguide atop an oxide pillar, while the base corresponds to bulk silicon that has been micromachined. The thick rectangles indicate the metal waveguide structure, made of copper.
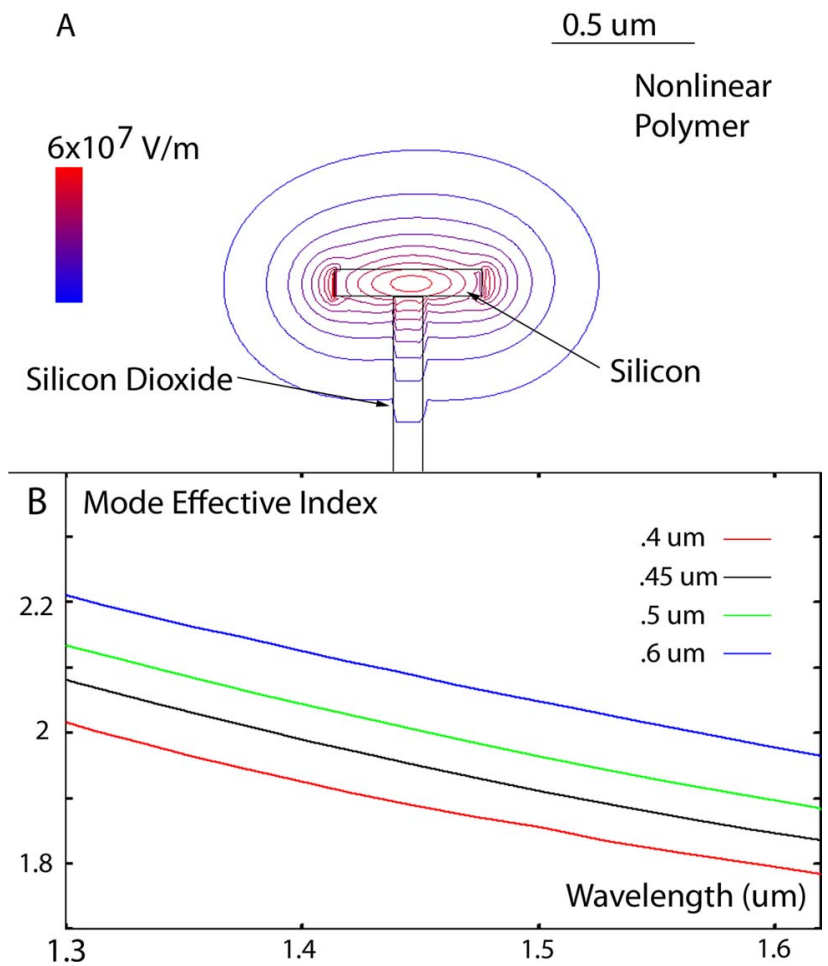

Fig. 2. (Color online) Diagram of the optical waveguide. Diagram is shown in panel A, with contours of the mode rendered in increments of $10 \%$ of $|E|$ assuming $1 \mathrm{~W}$ of input power. The waveguide used was $500 \mathrm{~nm}$ wide and $100 \mathrm{~nm}$ tall; the width of this waveguide is varied as a design parameter. The Silicon wire waveguide is supported by a $100 \mathrm{~nm}$ wide pillar of $\mathrm{SiO}_{2}$. In panel $\mathrm{B}$, the dispersion diagrams of several waveguide widths are plotted.

guide. The effective indices of refraction from three different $\mathrm{Si}$ waveguide widths are presented in panel $\mathrm{B}$. The modes were solved for using a Hermetian eigensolver [14]. With such guides, phase matching for $\mathrm{THz}$ modes with effective indices ranging from 2.8 to 3.2 is practical.

Similar optical waveguides have been shown to have losses of approximately $3.5 \mathrm{~dB} / \mathrm{cm}$ and are expected to improve significantly in the future [29]. From perturbation theory, the loss for the optical mode from interactions with the surrounding metal waveguide can be bounded to not exceed $3 \mathrm{~dB} / \mathrm{cm}$, and it is generally significantly less. As typical device lengths for the nonlinear interaction region are expected to be below $2.5 \mathrm{~mm}$, optical losses of even $10 \mathrm{~dB} / \mathrm{cm}$ would not present a limitation.

In designing the $\mathrm{THz}$ waveguide, we wished to achieve two goals. First, we wanted to make the THz mode more compact, since this maximizes the figure of merit, as can easily be seen from Eq. (15). Second, we wanted a waveguide that was relatively broadband. Whereas spanning as much as $10 \mathrm{THz}$ in the optical regime does not amount to a large fractional shift in frequency, the same is certainly not true in the $\mathrm{THz}$ regime. As a result, we sought a design inspired by a transmission line, since TEM modes often span frequency regimes of many orders of magnitude.

To span the maximum frequency range, two $\mathrm{THz}$ waveguide designs (labeled I and II) are presented. They are both similar to a conventional copper dual-strip transmis- 
Table 1. Description of Terahertz Waveguides and Device Performances ${ }^{a}$

\begin{tabular}{cllllll}
\hline $\begin{array}{c}\text { Terahertz } \\
\begin{array}{c}\text { Waveguide } \\
\text { Type }\end{array}\end{array}$ & $\mathrm{A}(\mu \mathrm{m})$ & $\begin{array}{l}\mathrm{B} \\
(\mathrm{um})\end{array}$ & $\begin{array}{l}\mathrm{C} \\
(\mathrm{um})\end{array}$ & $\begin{array}{l}\mathrm{D} \\
(\mathrm{um})\end{array}$ & $\begin{array}{l}\mathrm{E} \\
(\mathrm{um})\end{array}$ & $\begin{array}{l}\mathrm{F} \\
(\mathrm{um})\end{array}$ \\
\hline $\mathrm{I}$ & 14 & 3.5 & $>20$ & 2 & 2 & 2 \\
$\mathrm{II}$ & 4.7 & 3 & $>20$ & 1 & 1.3 & 2 \\
\hline
\end{tabular}

${ }^{a}$ Columns A through $\mathrm{F}$ correspond to the dimensions in Fig. 1. The data in column $\mathrm{C}$ refer to the fact that this dimension is to exceed the specified value. Label $\mathrm{G}$ is not present, as the optical waveguide width is varied independently.

sion line. However, the portion of the transmission line where the mode resides is partially filled with a plug of silicon, which is part of the supporting silicon pillar. The purpose of this feature is to raise the effective refractive index of the $\mathrm{THz}$ mode and to ensure that the phasematching condition is met. Precise dimensions of the two designs are given in Table 1.

The complex geometry of the $\mathrm{THz}$ guide makes mode solving challenging. The modes were solved by launching the dual-strip transmission line pattern into a full $3 \mathrm{D}$ simulation, and observing the field pattern at various points down the waveguide. A steady-state analysis was used on the Yee grid [30]. The discretization was $0.1 \mu \mathrm{m}$, and the run length was around $120 \mu \mathrm{m}$. From the relative uniformity and $z$ behavior of the fields so obtained, it was clear that this method yielded the accurate modes. Once the modal pattern is known, the losses and effective indices of the modes were easily calculated via the relevant closed-form expressions. Note that in the actual simulation, a perfect metal was assumed. However, using perturbation theory, the waveguide loss due to imperfect metals could then be calculated.

In Fig. 3, the modal patterns for $\mathrm{THz}$ waveguides I and II are shown at various frequency extrema. At higher frequencies, the $\mathrm{THz}$ mode tends to migrate into the region with more silicon. This has the unfortunate effect of pulling the field away from the optical waveguide and decreasing the generated power. The effective indices of the terahertz waveguides are shown in panel B of Fig. 4.

The waveguide loss of the $\mathrm{THz}$ mode is highly frequency dependent. This is due to the effect of increased frequency on Ohmic losses, as well as frequency dependence of the mode pattern The oxide pillar supporting the optical waveguide will not significantly impact the $\mathrm{THz}$ mode loss, since the relevant mode overlap is very small. Bulk polymer losses as high as $30 \mathrm{~dB} / \mathrm{cm}$ could be tolerated without significantly altering device performance. It is anticipated that future nonlinear polymers will easily meet this standard [16]. Substrate leakage is not expected to be a major issue, provided that the support pillars are $20 \mu \mathrm{m}$ or more in height. Even over 3-D simulations of $100 \mu \mathrm{m}$ or more of waveguide, no substrate leakage is evi-

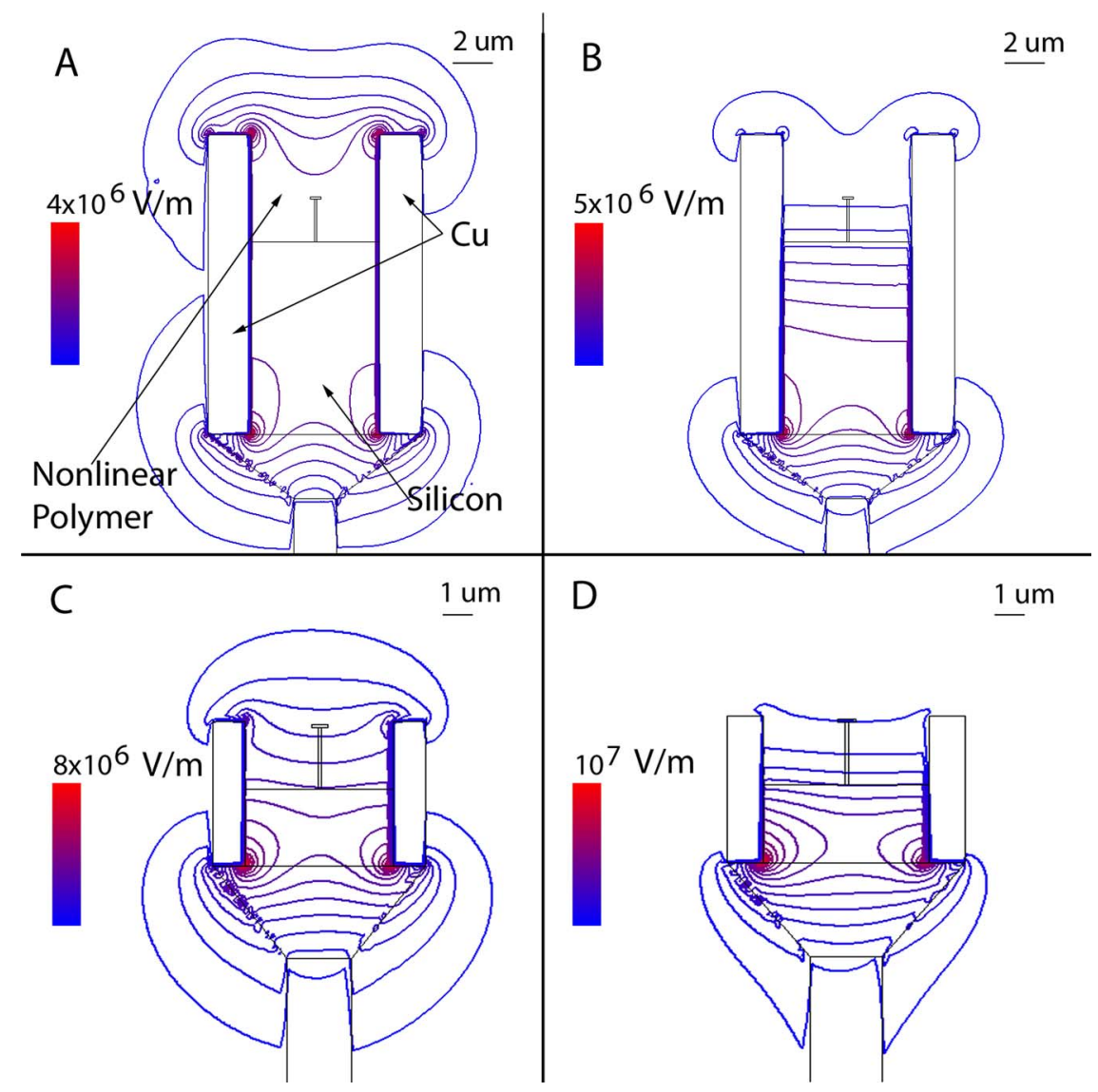

Fig. 3. (Color online) Terahertz waveguide modal patterns. The modal patterns for terahertz waveguide I is shown in panels A and B, at frequencies 1 and $6 \mathrm{THz}$, respectively, for $1 \mathrm{~W}$ of propagating power. The modal patterns for terahertz waveguide II are shown in panels $\mathrm{C}$ and $\mathrm{D}$ at 6 and $14 \mathrm{THz}$, respectively. In all cases, the $|E|$ field contours are plotted in increments of $5 \%$. The maximum field in Volts per meter is also indicated on a scale bar. This is for a mode with a time-average energy of $1 \mathrm{~W}$. 


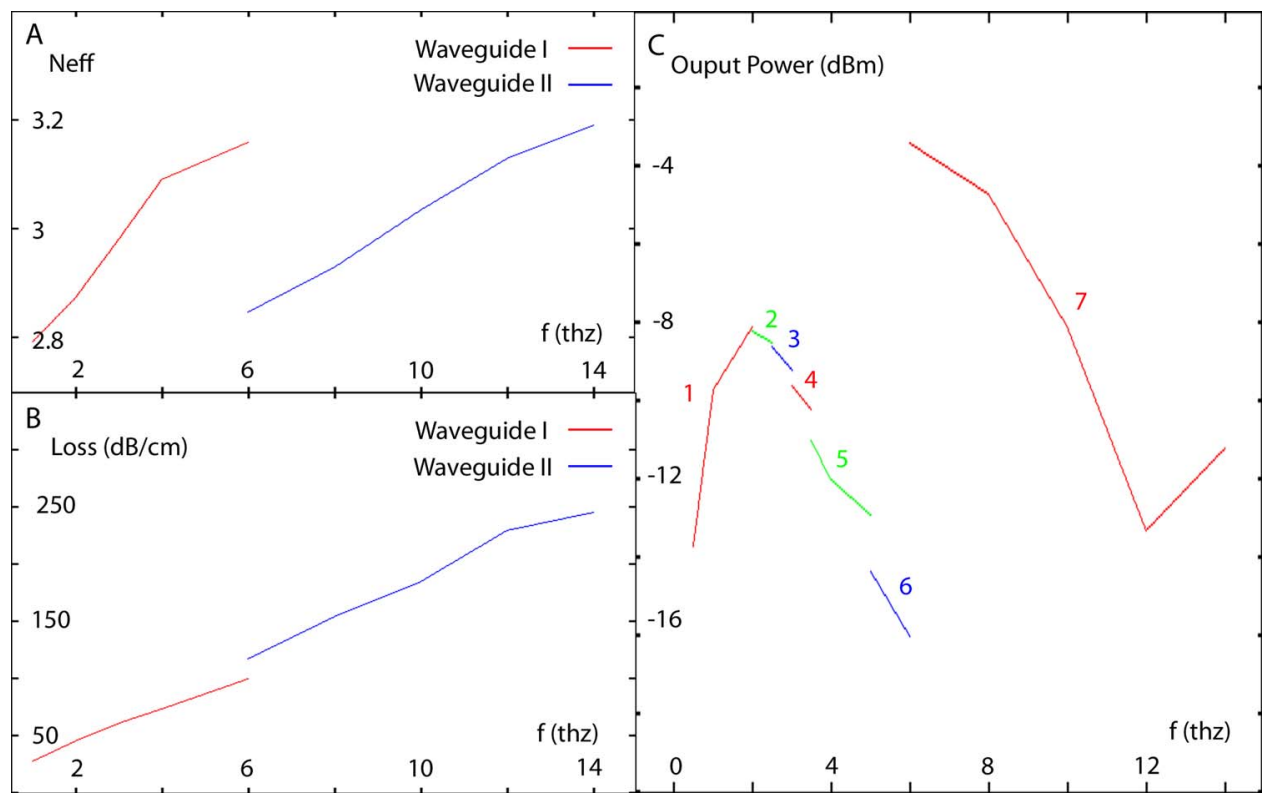

Fig. 4. (Color online) Characteristics of terahertz waveguide modes and output power. Panel A shows the effective index of the terahertz modes as a function of frequency. Panel B shows the waveguide loss in decibels per centimeter. In panel C, the output power is specified for devices specified in Table 1, in decibels of output power versus the output frequency in terahertz.

dent in such a configuration. While a small amount, perhaps less than $0.1 \mathrm{~dB}$, might remain undetected in these models, this still provides an upper bound of $10 \mathrm{~dB} / \mathrm{cm}$, which is far less than the losses due to other sources.

Once the effective indices for the modes are established, and the precise modal patterns are known, it is possible to calculate the conversion efficiency via the relations presented in Section 2. Table 2 and Fig. 4 provide a summary of the cw output powers achievable as a function of wavelength. It is possible to change the waveguide width lithographically, suggesting that these devices may well serve in an array to maximize the frequency coverage on a particular chip. Output power in the high microwatt level is predicted across the entire frequency range from $0.5 \mathrm{THz}$ up to $15 \mathrm{THz}$.

An important characteristic of these devices is that the generated $\mathrm{THz}$ beam can be interchanged with one of the optical beams. This can be seen by noting that Eqs. (9) and (10) are symmetric in the electric fields of all waveguides, to a complex conjugation and a frequency factor in front. As a result, if two optical beams at 0.5 watts each generate a $1 \mathrm{THz}$ output of $0.5 \mathrm{~mW}$, then an optical beam at $0.5 \mathrm{~W}$ and a $\mathrm{THz}$ beam at $0.5 \mathrm{~mW}$ will generate an optical beam of $18 \mathrm{~mW}$ in the exact same de-

Table 2. Full Device Designs with Their Performance Data ${ }^{a}$

\begin{tabular}{|c|c|c|c|c|c|c|}
\hline $\begin{array}{c}\text { Device } \\
\text { No. }\end{array}$ & $\begin{array}{c}\text { Optical } \\
\text { Waveguide } \\
\text { Width } \\
(\mu \mathrm{m})\end{array}$ & $\begin{array}{l}\text { Device } \\
\text { Length } \\
(\mathrm{mm})\end{array}$ & $\begin{array}{c}\text { Terahertz } \\
\text { Waveguide } \\
\text { Type }\end{array}$ & $\begin{array}{l}\text { Output } \\
\text { Frequency } \\
\text { Range } \\
(\mathrm{THz})\end{array}$ & $\begin{array}{l}\text { Output } \\
\text { Power } \\
\text { Range } \\
(\mathrm{dBm})\end{array}$ & $\begin{array}{c}\text { Input } \\
\text { Laser } \\
\text { Range } \\
\text { Needed } \\
\text { (nm) }\end{array}$ \\
\hline 1 & 0.375 & 5 & I & $0.5-2$ & $-13.7,-8.1$ & $\begin{array}{c}1480- \\
1600\end{array}$ \\
\hline 2 & 0.4 & 4 & I & $2-2.5$ & $-8.2,-8.5$ & $\begin{array}{c}1480- \\
1600\end{array}$ \\
\hline 3 & 0.425 & 3 & I & $2.5-3$ & $-8.6,-9.2$ & $\begin{array}{c}1480- \\
1600\end{array}$ \\
\hline 4 & 0.45 & 3 & I & $3-3.5$ & $-9.6,-10.2$ & $\begin{array}{c}1480- \\
1600\end{array}$ \\
\hline 5 & 0.5 & 3 & I & $3.5-5$ & $-11,-12.9$ & $\begin{array}{c}1480- \\
1600\end{array}$ \\
\hline 6 & 0.6 & 2.5 & I & $5-6$ & $-14.3,-16$ & $\begin{array}{c}1480- \\
1600\end{array}$ \\
\hline 7 & 0.4 & 2 & II & $6-14$ & $-3.4,-11.2$ & $\begin{array}{c}1300- \\
1600\end{array}$ \\
\hline
\end{tabular}

${ }^{a}$ The two input lasers are assumed to be $500 \mathrm{~mW}$ in cw power. The $\chi^{2}$ value is taken to be $500 \mathrm{pm} / \mathrm{V}$, or $2 \times 10^{-9} \mathrm{~m} / \mathrm{V}$. The optical waveguide loss is taken to be $6 \mathrm{~dB} / \mathrm{cm}$, which is a safe upper bound on expected losses. Note that the optical waveguide width corresponds to letter G in Fig. 1 . The predicted output spectra generated by these devices are plotted in Fig. 4. 
vice. That is, an STG can be used to shift a $\mathrm{THz}$ signal into the optical regime and also add about $15 \mathrm{~dB}$ of power. The output beam will be located at the pump optical frequency plus the $\mathrm{THz}$ frequency, allowing for $\mathrm{THz}$ detection schemes that will use conventional diode- or photomultiplier-tube-based optical detectors, which are extremely sensitive.

\section{HEATING AND FREE-CARRIER LIMITATIONS ON PERFORMANCE}

A typical problem with integrated optical CW devices is the dissipation of waste heat. Clearly, any form of loss that does not result in scattered radiation will eventually convert the optical input energy to heat. Under continuous operation, this heat source will raise the temperature of the waveguide and polymer until it is balanced by heat dissipation mechanisms. If the steady-state temperature exceeds a damage threshold, the device will fail.

A thorough discussion of the extraction of waste heat, and the limitations this will impose on $\mathrm{cw}$ input laser power, is beyond the scope of this paper. Also, such considerations will be driven in part by the precise characteristics of the polymer, parameters that can be engineered with this specific function in mind. However, we are optimistic that the power levels we have described could be supported. We have already demonstrated that cw optical power levels of $100 \mathrm{~mW}$ can be supported in such waveguides clad with nonlinear polymers [22]. Typically, damage thresholds are encountered a few decibels beyond this level, at around $200 \mathrm{~mW}$. To support the two input lasers of $0.5 \mathrm{~W}$ that we have proposed above, the damage threshold would need to be increased by less than 1 order of magnitude, which suggests it is achievable with careful engineering.

Another consideration is the fact that even though radiation at a $1300 \mathrm{~nm}$ wavelength and longer is beyond the silicon band edge, various mechanisms can produce free carriers, especially at high power. One such mechanism is two-photon absorption (TPA) [31]. In very small silicon ridge waveguides such as the ones we propose to use, surface-state absorption can also create free carriers [32]. This creates nonlinear loss on the optical signal, which can lead to rapid signal attenuation. To determine whether this would be a limitation, nonlinear loss measurements were performed on silicon-on-insulator waveguides with the $0.5 \times 0.1 \mu \mathrm{m}$ dimension given above. It was found that the loss within a waveguide was well modeled by

$$
\frac{\mathrm{d} I}{\mathrm{~d} z}=-\alpha_{1} I-\alpha_{2} I^{2}
$$

where $I$ is the propagating cw intensity of a beam, and $\alpha_{1}$ is $0.00014 \mu \mathrm{m}^{-1}$, while $\alpha_{2}$ was $1.8 \times 10^{-6} \mu \mathrm{m}^{1} \mathrm{mw}^{-1}$. This corresponds to about $12 \mathrm{~dB} / \mathrm{cm}$ of additional loss at a $\mathrm{cw}$ power of $150 \mathrm{~mW}$. While this is certainly problematic, it should be remembered that the $\mathrm{THz}$ waveguide losses are far higher, and so the interaction length in which a $\mathrm{THz}$ beam can be generated is fairly short. Therefore, the optical losses are not important in most cases until they cause significant attenuation in only a few millimeters.
All-optical pump-probe measurements were also performed in order to determine minority carrier lifetimes; this work will also be detailed in a forthcoming publication [32]. The minority carrier lifetime was found to be around $1 \mathrm{~ns}$, which can be compared to the typical values of 1-10 ns reported by Jalali [33].

The nonlinear loss can be reduced by removing the free carriers. Several methods have been demonstrated for electrically sweeping excess carriers from a silicon waveguide to reduce effective carrier lifetime [34,35]. Additionally, it has been demonstrated that ion implantation of $\mathrm{Ar}^{+}$ions can reduce the free-carrier lifetime to $100 \mathrm{ps}$ [36], which would amount to a decrease in the nonlinear loss that our waveguides experience by a factor of 10 . This approach could also be easily integrated into our process, since it does not require any change in the waveguide geometry or electrical contact. Finally, it is possible to treat silicon waveguides such as these to reduce surface-state absorption and waveguide loss [37]. Lowering the waveguide loss would also help to raise the thermal damage threshold.

Absorption of the $\mathrm{THz}$ mode by free carrier accumulation is expected to be less important. This is due to the fact that though the absorption coefficient of $\mathrm{THz}$ radiation due to free carriers is approximately 100 times larger than the absorption coefficient at $1550 \mathrm{~nm}$ [38], the area of the silicon waveguide is around $1 \%$ of the area of the $\mathrm{THz}$ mode, suggesting that the $\mathrm{THz}$ mode will not experience larger losses than the optical mode due to a given free-carrier concentration. Of course, the $\mathrm{THz}$ mode already experiences losses that are much higher than the optical signal to begin with, so it would take a proportionally larger amount of loss to seriously degrade device performance.

While free-carrier losses would be an issue in using the current silicon ridge waveguides in a device, the current nonlinear loss is within 1 order of magnitude of what would be needed for the proposed designs. Furthermore, there are numerous examples in the literature of solutions to this problem. It is therefore likely that with sufficient engineering, optically induced free-carrier absorption does not pose a fundamental limitation to the proposed device.

\section{CONCLUSIONS}

We have presented a design for a device that, if realized, would allow the room temperature cw generation of $\mathrm{THz}$ radiation. Other than the nonlinear polymer, our system consists of materials that are commonly used in semiconductor manufacturing processes. We have also shown that the two most important obstacles to high-power performance, thermal damage and optically free-carrier excitation, are likely to be surmountable with a reasonable level of engineering. Our STG can function at room temperature and can provide a level of output power that is far larger than what comparable processes can currently demonstrate. We believe that our device, once realized, has the potential to make the $\mathrm{THz}$ regime vastly more accessible to science and industry. 


\section{ACKNOWLEDGMENT}

This work was supported in part by the Air Force Office of Scientific Research (FA9550-04-1-0434) and DARPA (HR0011-04-1-0054).

\section{REFERENCES}

1. E. Mueller, "Terahertz radiation sources for imaging and sensing applications," Photonics Spectra 40, 60 (2006).

2. M. Hoheisel, "Review of medical imaging with emphasis on X-ray detectors," Nucl. Instrum. Methods Phys. Res. A 563, 215-224 (2006)

3. D. L. Woolard, E. R. Brown, M. Pepper, and M. Kemp, "Terahertz frequency sensing and imaging: a time of reckoning future applications?," Proc. IEEE 93, 1722-1743 (2005).

4. A. V. Quema, M. Goto, M. Sakai, R. E. Oenzerfi, H. Takahashi, H. Murakami, S. Ono, N. Sarukura, and G. Janairo, "Onset detection of solid-state phase transition in estrogen-like chemical via terahertz transmission spectroscopy," Appl. Phys. Lett. 85, 3914-3916 (2004).

5. K. J. Linden, W. R. Neal, J. Waldman, A. J. Gatesman, and A. Danylov, "Terahertz laser based standoff imaging system," in 34th Applied Imagery Pattern Recognition Workshop (Applied Imagery Pattern Recognition, 2005), pp. 7-14.

6. Bruker Optics, http://www.brukeroptics.com.

7. Y. Sasaki, A. Yuri, K. Kawase, and H. Ito, "Terahertz-wave surface-emitted difference frequency generation in slantstripe-type periodically poled $\mathrm{LiNbO}_{3}$ crystal," Appl. Phys. Lett. 81, 3323-3325 (2002).

8. V. Kukushkin, "Efficient generation of terahertz pulses from single infrared beams in $\mathrm{C} / \mathrm{GaAs} / \mathrm{C}$ waveguiding heterostructures," J. Opt. Soc. Am. B 23, 2528-2534 (2006).

9. J. Faist, F. Capasso, D. L. Sivco, C. Sirtori, A. L. Hutchinson, and A. Y. Cho, "Quantum cascade laser," Science 264, 553-556 (1994).

10. Virginia Diodes, Inc., http://virginiadiodes. com/.

11. M. A. Belkin, F. Capasso, A. Belyanin, D. L. Sivco, A. Y. Cho, D. C. Oakley, C. J. Vineis, and G. W. Turner, "Terahertz quantum-cascade-laser source based on intracavity difference-frequency generation," Nat. Photonics 1, 288-292 (2007).

12. H. T. Chen, W. J. Padilla, J. M. O. Zide, A. C. Gossard, A. J. Taylor, and R. D. Averitt, "Active terahertz metamaterial devices," Nature 444, 597-600 (2006).

13. M. Tonouchi, "Cutting-edge terahertz technology," Nat. Photonics 1, 97-105 (2007).

14. T. Baehr-Jones, M. Hochberg, C. Walker, and A. Scherer, "High- $Q$ resonators in thin silicon-on-insulator," Appl. Phys. Lett. 85, 3346-3347 (2004).

15. Y. Enami, C. T. Derose, D. Mathine, C. Loychik, C. Greenlee, R. A. Norwood, T. D. Kim, J. Luo, Y. Tian, A. K. Y. Jen, and N. Peyghambarian, "Hybrid polymer/sol-gel waveguide modulators with exceptionally large electrooptic coefficients," Nat. Photonics 1, 180-185 (2007).

16. L. R. Dalton, Chemistry Department, University of Washington, Box 351700, Seattle, Wash, 98195 (personal communication, 2006).

17. A. Yariv, Quantum Electronics (Wiley, 1989).

18. T. Baehr-Jones, M. Hochberg, G. X. Wang, R. Lawson, Y. Liao, P. A. Sullivan, L. Dalton, A. K. Y. Jen, and A. Scherer, "Optical modulation and detection in slotted silicon waveguides," Opt. Express 13, 5216-5226 (2005).

19. J. D. Jackson, Classical Electrodynamics (Wiley, 1998).

20. T. Baehr-Jones, M. Hochberg, C. Walker, E. Chan, D. Koshinz, W. Krug, and A. Scherer, "Analysis of the tuning sensitivity of silicon-on-insulator optical ring resonators," J. Lightwave Technol. 23, 4215-4221 (2005).

21. E. Palik, Handbook of Optical Constants of Solids (Academic, 1985).

22. M. Hochberg, T. Baehr-Jones, G. X. Wang, M. Shearn, K. Harvard, J. D. Luo, B. Q. Chen, Z. W. Shi, R. Lawson, P. Sullivan, A. K. Y. Jen, L. Dalton, and A. Scherer, "Terahertz all-optical modulation in a silicon-polymer hybrid system," Nat. Mater. 5, 703-709 (2006).

23. D. Taillaert, W. Bogaerts, P. Bienstman, T. F. Krauss, P Van Daele, I. Moerman, S. Verstuyft, K. De Mesel, and R. Baets, "An out-of-plane grating coupler for efficient buttcoupling between compact planar waveguides and singlemode fibers," IEEE J. Quantum Electron. 38, 949-955 (2002).

24. A. S. Liu, R. Jones, L. Liao, D. Samara-Rubio, D. Rubin, O. Cohen, R. Nicolaescu, and M. Paniccia, "A high-speed silicon optical modulator based on a metal-oxidesemiconductor capacitor," Nature 427, 615-618 (2004).

25. M. Lipson, "Overcoming the limitations of microelectronics using Si nanophotonics: solving the coupling, modulation and switching challenges," Nanotechnology 15, S622-S627 (2004).

26. J. Plant, P. W. Juodawlkis, R. K. Huang, J. P. Donnelly, L. J. Missaggia, and K. G. Ray, "1.5- $\mu \mathrm{m}$ InGaAsP-InP slabcoupled optical waveguide lasers," IEEE Photonics Technol. Lett. 17, 735-737 (2005).

27. Calmar Optcom, http://www.calmaropt.com.

28. H. Tazawa, Y. H. Kuo, I. Dunayevskiy, J. D. Luo, A. K. Y. Jen, H. R. Fetterman, and W. H. Steier, "Ring resonatorbased electro-optic polymer traveling-wave modulator," J. Lightwave Technol. 24, 3514-3519 (2006).

29. M. Borselli, T. J. Johnson, and O. Painter, "Beyond the Rayleigh scattering limit in high- $Q$ silicon microdisks: theory and experiment," Opt. Express 13, 1515-1530 (2005).

30. A. Taflove and S. Hagness, Computational Electrodynamics (Artech House, 2000).

31. D. Dimitropoulos, S. Fathpour, and B. Jalali, "Limitations of active carrier removal in silicon Raman amplifiers and lasers," Appl. Phys. Lett. 87, 261108 (2005).

32. T. Baehr-Jones, M. Hochberg, and A. Scherer are preparing a manuscript to be called "Photodetection in silicon beyond the band edge with surface states."

33. B. Jalali, "Teaching silicon new tricks," Nat. Photonics 1, 193-195 (2007).

34. S. F. Preble, Q. Xu, B. Schmidt, and M. Lipson, "Ultra-fast all-optical modulation on a silicon chip," Opt. Lett. 30, 2891-2893 (2005)

35. H. Rong, Y. H. Kuo, S. Xu, A. Liu, R. Jones, and M. Paniccia, "Monolithic integrated Raman silicon laser," Opt. Express 14, 6705-6712 (2006).

36. T. Tanabe, K. Nishiguchi, A. Shinya, E. Kuramochi, H. Inokawa, and M. Notomi, "Fast all-optical switching using ion-implanted silicon photonic crystal nanocavities,” Appl. Phys. Lett. 90, 031115 (2007).

37. M. Borselli, "High- $Q$ microresonators as lasing elements for silicon photonics," Ph.D. thesis (California Institute of Technology, 2006).

38. R. Soref and B. Bennett, "Electro-optical effects in silicon," IEEE J. Quantum Electron. 23, 123-129 (1987) 